\title{
Effects of 2-Deoxy-D-Glucose, D-Glucosamine, and L-Sorbose on the Growth of Coprinus lagopus hyphae
}

\author{
By D. MOORE AND G. R. STEWART \\ Department of Botany, The University, Manchester, $M{ }_{13} 9 P L$ \\ (Accepted for publication 3 February 1972) \\ SUMMARY
}

\begin{abstract}
2-Deoxy-D-glucose (deGlc), D-glucosamine (GA) and L-sorbose (Sor) inhibited extension of Coprinus lagopus hyphae; deGlc was about four times more inhibitory than GA, while the latter was about five times more inhibitory than Sor. The inhibitory effects were not uniformly additive. There were indications that the analogues competed with one another, suggesting they shared at least one point of action. Acetate and fructose were virtually ineffective in reversing the inhibitory effect. Glucose was ro times more effective than mannose and about 100 times more effective than fructose or acetate in reversing inhibition. The fact that the analogues inhibited most on media which either lacked normal carbon source or which contained acetate was interpreted as indicating that growth-rate inhibitions which occurred on media containing hexose sugar did not result from any significant effect on sugar transport.

All three analogues caused gross morphological changes. The most characteristic aberration was a distinct swelling of the hyphal tip. This was most severe with GA which caused the tip cell to balloon into a spheroid some five to six times the diameter of the subtending hypha. The most extreme consequence of swelling occurred on media containing deGlc; after swelling to two to three times their normal diameters, tips frequently became vacuolated and often burst. Tip lysis was not observed on media containing either GA or Sor.
\end{abstract}

\section{INTRODUCTION}

2-Deoxy-D-glucose (deGlc) severely inhibited the germination of the haploid, asexual spores (oidia) of Coprinus lagopus but the spores were not killed (Moore, I968). The extent of the inhibition depended not only on the ratio of normal hexose to analogue, but also on the identity of the normal hexose. Glucose was at least Ioo times more effective than fructose in reversing the inhibition. The inhibitory effect of deGlc on oxygen uptake by germinating oidia followed fairly closely the inhibitions of germination and hyphal extension. Oxygen uptake was still measurable on inhibitor-media which completely inhibited germ-tube and hyphal growth.

Heredia, De la Fuente \& Sols (1964) showed that the deGlc-mediated inhibition of the growth of Saccharomyces cerevisiae was much greater than inhibition of glycolysis. Such results suggest that the total deGlc inhibition follows from the accumulation of a number of lesser inhibitory actions.

A detailed examination of the effects of L-sorbose (Sor) on the extension growth of Coprinus was prompted by the finding that mutants selected for resistance to the inhibitory effects of deGlc were cross-resistant to sorbose inhibition. The later demonstration that these same mutants were also cross-resistant to inhibitions caused by D-glucosamine (GA) led directly to the inclusion of this chemical in the analysis. 
Barnett \& Lilly (195I) showed that the growth of many fungi was inhibited by Sor, although they later showed that some fungi were able to utilize the hexose either as sole carbon source or synergistically in the presence of glucose or maltose (Lilly \& Barnett, I953). The same spectrum of Sor effects (complete inhibition in some cases, ability to utilize singly or in combination with more normal carbon sources in other cases) has been recorded in different genera of Basidiomycetes (Oddoux, I952), different species of Phyllosticta (Bilgrami, I963), and different monosporidial isolates of Ustilago maydis (Matsushima \& Klug, 1958).

Although a great deal is known about the physiological and enzymatic effects of glucosamine in animal tissues, and to some extent in bacteria, it does not appear to have been recognized as an inhibitor of fungal growth. It may be that the importance of the glucosamine structure to the construction of fungal hyphal walls has diverted attention from these other properties of GA.

\section{METHODS}

Organism. The strain used for the major part of the work was the backcrossed monokaryotic wild-type strain, stock number $\mathrm{BC} 9 / 6,6$ (Moore, I969 $b$ ). Five strains isolated from the wild have also been used. These were: zBw60I/40, 40, a monokaryon derived from a dikaryon supplied by J. Necasek of the Karlovy University, Prague, Czechoslovakia; and strains BI, HI, 2HI and PA2 - monokaryons isolated by H. Waters from vegetative stocks and silica-gel spore preparations supplied by P. R. Day of the Connecticut Agricultural Experiment Station, New Haven, U.S.A., and by D. H. Morgan of the John Innes Institute, Norwich, Norfolk.

Media. The basal medium used was the fully defined NCM medium (Moore, $1969 b$ ) with

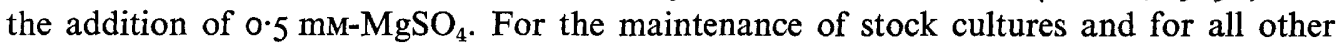
general purposes the complete medium (CM) described by Moore (1968) was used. Media were solidified with $\mathrm{I} \cdot 5 \%$ (w/v) Difco Bacto-Agar. Carbon sources were either prepared in the form of stock solutions (usually $0.5 \mathrm{M}$ ), sterilized with chloroform and then pipetted in appropriate quantity to the previously autoclaved ( $15 \mathrm{lb} / \mathrm{in}^{2}$ for $15 \mathrm{~min}$ ) basal medium; or were added as the solid to the autoclaved basal medium without attempt at sterilization. Glaxo 'Crystapen' benzylpenicillin was added to all media to a final concentration of 80 to Ioo i.u./ml. 2-Deoxy-D-glucose was obtained from the Sigma Chemical Company, London. Other chemicals, of Analar grade where possible, were obtained from BDH Chemicals Limited, Poole, Dorset. The fructose used was the preparation described as 'glucose-free' by the manufacturers.

Assessment of linear growth. Media were poured into glass Petri dishes (about $25 \mathrm{ml} / \mathrm{dish}$ ) and inoculated centrally with $2 \mathrm{~mm}$ diameter discs cut from the margins of six-day-old colonies growing on CM. Four replicates were made of each test. Colonies were measured across two diameters at right-angles, usually at about $24 \mathrm{~h}$ intervals, for a period of five days. All incubations were made at $37^{\circ} \mathrm{C}$. Plots of colony radius against time were usually found to be linear. In these cases growth rates were determined by calculating the constants of the regression equation for each set of data.

Microscopic observations. Hyphal morphology was studied by transferring pieces of agar with their attached hyphae to microscope slides. The mycelium was stained with Cotton Blue in lactophenol and photographed with a Reichert 'Zetopan' microscope. 
Table 1. Comparison between the linear growth rate of Coprinus lagopus and the yield of mycelium produced on media containing different initial concentrations of carbon source

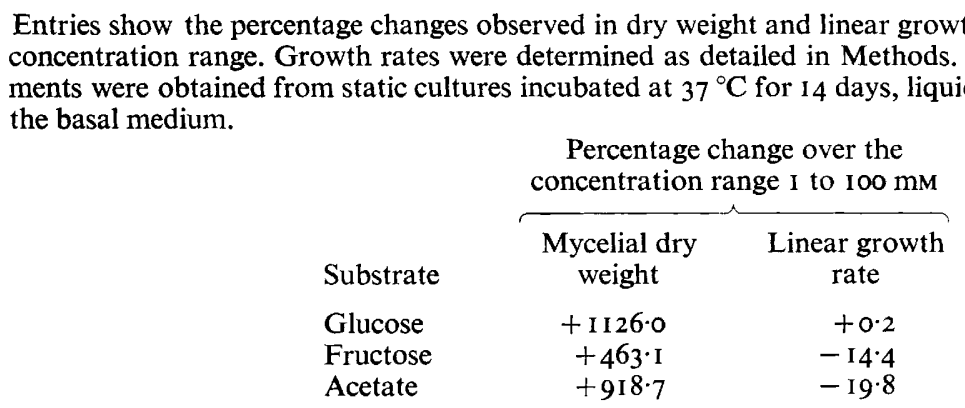

RESULTS

\section{Linear growth as a reliable parameter}

The extent and rates of linear growth were the only criteria of the gross physiological effects of the inhibitors used in this study. The data in Table I demonstrate that linear growth is, in Coprinus, a very reliable parameter. Over a considerable concentration range of normal carbon sources there was very little change in the rate of linear growth although the growth yield in terms of dry weight of mycelium obtained from liquid cultures increased dramatically. This lack of correlation between linear growth rates and the mass of fungus produced is not uncommon in fungi. It appears to be caused by a number of factors, among them being the amount of hyphal branching and the growth rates of the branches (Mandels, 1965). Trinci (1969) showed that there was an inverse relationship between radial growth rate and hyphal density in colonies of Aspergillus nidulans grown on various concentrations of glucose. Since the linear growth of Coprinus was so little changed over the concentration range of carbon source shown in Table $\mathrm{I}$ we conclude that this parameter may be used with confidence as a measure of the effects of factors which tend to limit extension of the colony margin. This was certainly the conclusion of Trinci \& Gull (1970) from their study of the effects of three inhibitors on the growth kinetics of Aspergillus nidulans and Absidia glauca. In effect we have used linear growth rate as an assay system which was independent of those other factors which contribute to the specific growth rate.

\section{Effects of deGlc on linear growth of different strains of Coprinus}

The response of five strains to media containing deGlc were determined. The general pattern of inhibition was similar for all the strains.

\section{Effects of single inhibitors in media containing normal hexoses}

The inhibition of growth rate caused by increasing the concentration of each inhibitor was investigated in two ways. In the first type of experiment the concentration of inhibitor was increased while that of the normal carbon source was maintained at $5 \mathrm{~mm}$ (Fig. I). In the second type of experiment the ratio between the concentrations of normal carbon source and inhibitor was maintained at a predetermined value while the concentrations were increased (Fig. 2).

The results were very reproducible except when mannose was used as the normal carbon source. The data indicate that the response pattern on mannose-based media paralleled 


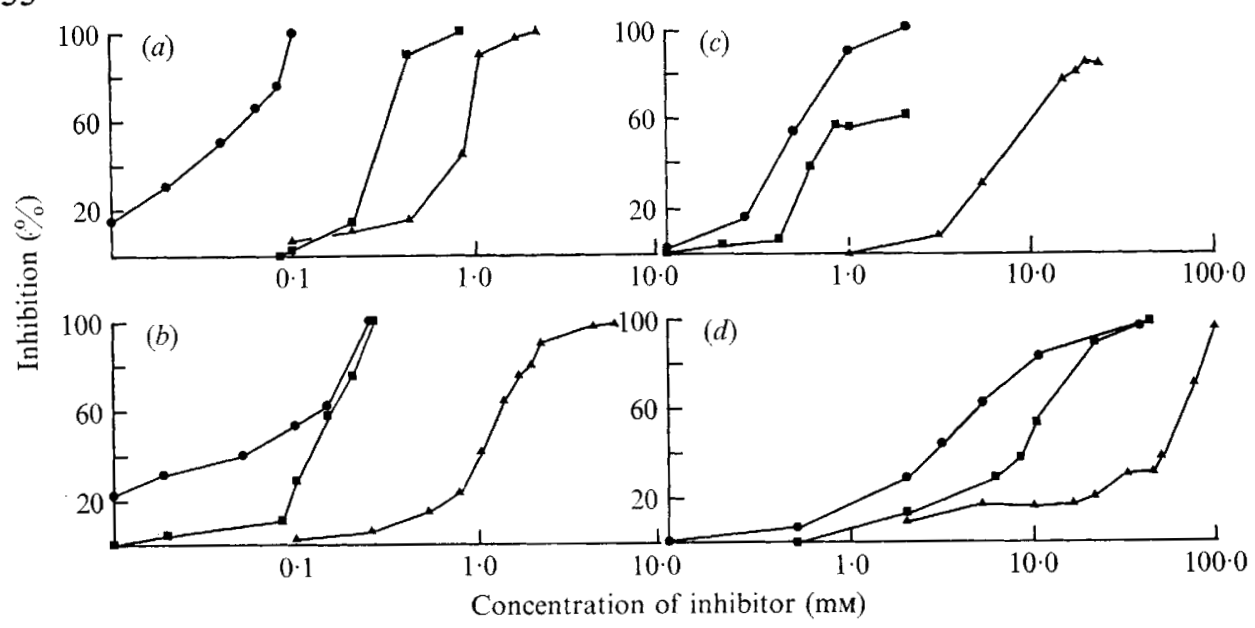

Fig. I. Inhibitory action of 2-deoxy-D-glucose (⿻), D-glucosamine ( $\boldsymbol{(})$ and L-sorbose $(\boldsymbol{\Delta})$ on the linear growth rate of Coprinus lagopus. (a) NCM basal medium containing no normal carbon source; (b) NCM basal medium containing $5 \mathrm{mM}$-fructose; (c) NCM basal medium containing 5 mM-mannose; $(d)$ NCM basal medium containing 5 mM-glucose. Ordinate: percentage inhibition of growth rate, arithmetic scale; abscissa: millimolar concentration of analogue, logarithmic scale.
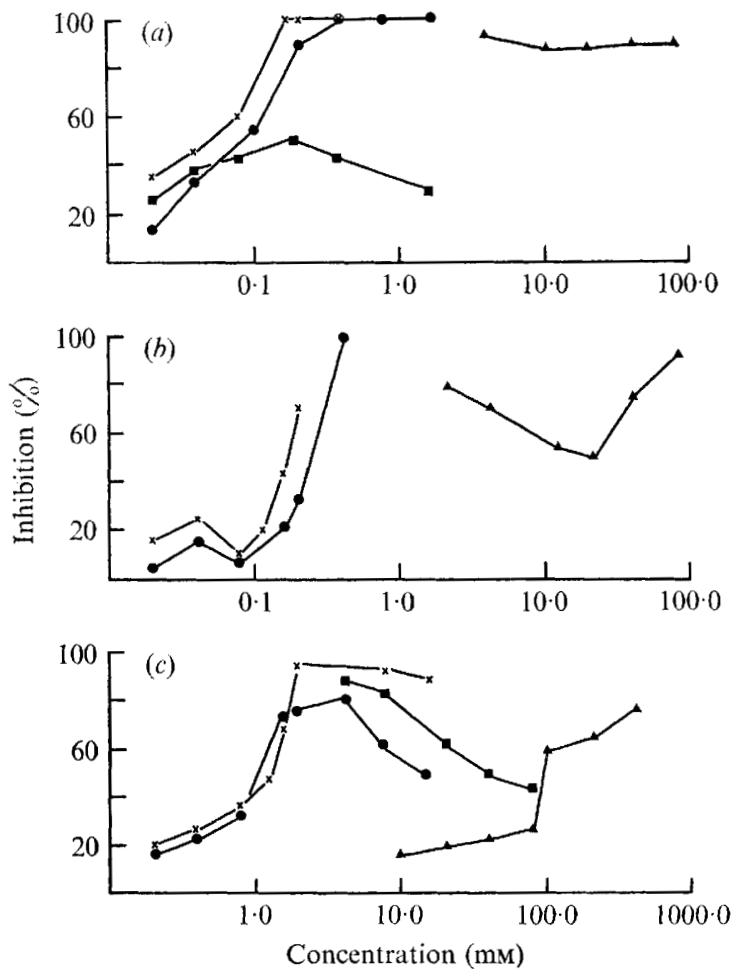

Fig. 2. Effect on the growth rate of Coprinus lagopus of increasing the concentration of each analogue while maintaining a constant ratio between the concentrations of inhibitor and of normal carbon source. (a) 2-Deoxy-D-glucose used at a constant $\mathrm{I}: 50$ ratio with sodium acetate $(x)$; fructose $(\boldsymbol{O})$ and mannose $(\boldsymbol{\square})$; and at a $2:$ I ratio with glucose $(\boldsymbol{\Delta})$. (b) D-Glucosamine used at a constant $\mathrm{I}: 50$ ratio with sodium acetate $(\times)$; and with fructose $(\bullet)$; and at a $2: 1$ ratio with glucose (A). (c) L-Sorbose used at a constant $I: 5$ ratio with sodium acetate $(x)$ and fructose $(\theta)$; at a $2: 1$ ratio with mannose (ם); and at a I0: 1 ratio with glucose (A). Ordinate: percentage inhibition of growth rate, arithmetic scale; abscissa: millimolar concentration of analogue, logarithmic scale. 
that on glucose media. In five different experiments in which inocula were transferred to $5 \mathrm{~mm}$-mannose the growth rates were I8I, I86, 290, 300 and II $7 \mu \mathrm{m} / \mathrm{h}$; whilst on $5 \mathrm{~mm}$ glucose the growth rates were $378,366,370,350$ and $37 \mathrm{I} \mu \mathrm{m} / \mathrm{h}$. These differences occurred despite the fact that tests carried out at the same time always used inocula from the same parental colony and were incubated for the same time in one incubator. The reasons for the variations are obscure, particularly since the standard deviations of the radii of replicates in any one experiment were never very marked. Colonies grown on mannose were morphologically different from those grown on any other normal carbon source, especially in having very little aerial mycelium. Strains BI, BC9/6,6 and 2HI had considerably slower extension rates on mannose than on the other carbon sources or when a carbon source was lacking. Strains HI, PA2 and ZBw60I/40,40 showed no such difference. These data may well be indicative of a population polymorphism with respect to mannose utilization.

\section{Effects of single inhibitors in media containing sodium acetate}

Acetate media frequently gave non-linear plots of colony radius against time. It was often observed that on media causing up to about $50 \%$ inhibition of growth rate an acceleration of growth occurred, in all replicates, after a considerable period of incubation. On media causing 50 to $100 \%$ inhibition such accelerations occurred less often, less consistently from trial to trial, and usually did not affect all of the replicates.

At low concentrations of inhibitor such effects may be explained, at least in part, in terms of adaptation to acetate utilization and it is clear that at low concentrations all three inhibitors either introduced a lag period or greatly extended an existing one. This can be understood on the assumption that the analogues tended to prevent in some way the successful induction of enzymes necessary for the utilization of acetate.

The occasional acceleration in growth observed on media causing more than $50 \%$ inhibition of extension rate was found to be due to the development of resistant sectors. The frequent occurrence of resistant sectors on acetate + inhibitor media, far more frequent than on any hexose + inhibitor medium, is difficult to understand. Data from experiments in which mutants resistant to deGlc were specifically selected showed that there was no corresponding difference in mutation frequencies of oidia plated on either acetate- or fructose-based media after mutagenesis. Thus a mean of 27.5 resistants were isolated per $10^{8}$ mutagen-treated oidia plated on fructose + deGlc while the mean yield from platings on acetate + deGlc was $23^{\circ} 9$ resistants per $10^{8}$ treated spores. It would seem, therefore, that the peculiar selective conditions which resulted in the appearance of so many resistant sectors in the growth rate experiments were restricted to hyphae.

Growth rate inhibitions caused by the analogues in acetate media are shown in Fig. 3 . The difficulties in establishing these inhibitions must be borne in mind when interpreting the data since acceleration in growth rate, whether caused by adaptation or mutation, would lead to underestimates of the degree of sensitivity.

Table 2 shows the approximate millimolar concentrations of each analogue which caused $50 \%$ inhibition of growth rate. deGlc had the greatest inhibitory effect and Sor the least. Glucose was the most effective in reversing the inhibitiosn, while fructose and acetate were the least effective.

\section{Effects of pair-wise mixtures of the analogues}

Table 3 shows growth rates of Coprinus lagopus on media containing mixtures of the three analogues. Interaction between the analogues is quite evident and though the position is obviously complicated two points stand out clearly. At very low, but not at intermediate 


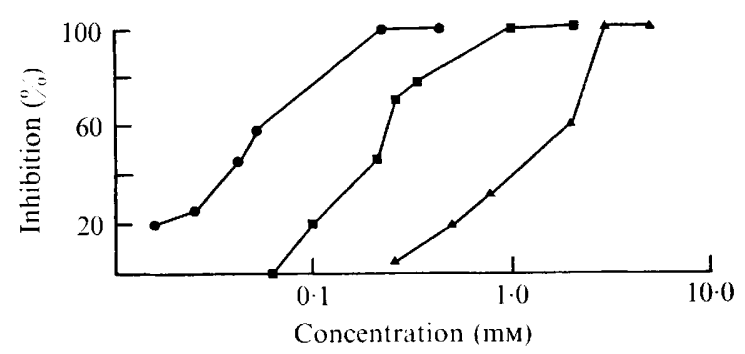

Fig. 3. Effect of 2-deoxy-D-glucose (O), D-glucosamine ( $\boldsymbol{\Pi})$, and L-sorbose $(\boldsymbol{\Delta})$ on the linear growth rate of Coprinus lagopus in medium containing $5 \mathrm{~mm}$-sodium acetate. Ordinate: percentage inhibition of growth rate, arithmetic scale; abscissa: millimolar concentration of analogue, logarithmic scale.

Table 2. Millimolar concentrations of each analogue which caused approximately $50 \%$ inhibition of linear growth rate

\begin{tabular}{lccc} 
Medium & \multicolumn{3}{c}{ Concentration of } \\
NCM alone & deGlc (mM) & GA (mM) & Sor (mM) \\
5 mM-Acetate & 0.04 & 0.30 & 0.80 \\
5 mM-Fructose & 0.05 & 0.20 & 1.50 \\
5 mM-Mannose & 0.08 & 0.20 & 1.00 \\
5 mM-Glucose & 0.40 & 0.70 & 9.00 \\
& 4.00 & 9.00 & 60.00
\end{tabular}

or high, analogue concentrations the inhibitions were more or less additive. A considerable degree of competition between the agents is indicated by these data.

\section{Effects on hyphal morphology}

The three analogues caused gross morphological changes in both the colony and its constituent hyphae. With increasing concentrations of inhibitor the normally radially symmetrical colonies became lobed and irregular in outline. All three agents caused a widening and shortening of intercalary and apical hyphal compartments (Fig. 4). GA caused the apical compartment to balloon into spheroids some five to six times the diameter of the sub-terminal cells. The most extreme consequence of swelling, however, occurred on media containing deGlc, for here the apical compartments, after swelling to about two to three times their normal diameter, frequently became vacuolated and often burst. Lysis of apical compartments was not observed on media containing either Sor or GA.

Recovery from swelling occurred but was variable. Terminal compartments affected by Sor almost always continued to grow, usually resuming growth through the (quite unusual) formation of multiple branches at the tip. Multiple branching of intercalary compartments was similarly observed on media containing inhibitory concentrations of Sor. Recovery from the effects of GA was also frequent, occurring through the outgrowth of single 'branches' from a region close to, but at one side of, the original tip of the apical compartment. The majority of apical compartments on media containing very inhibitory concentrations of deGlyc eventually lysed. At lower concentrations, however, some survived long enough for the formation of recovery branches; these again being formed eccentrically from a region immediately behind the extreme tip of the apical compartment. It was apparent, though, that such recovery from the effects of deGlc only postponed lysis, for on these media hyphae were regularly observed which consisted mainly of empty compartments, with just a 
Table 3. Growth rate inhibitions on media containing analogue mixtures

Medium constituents $\overbrace{\begin{array}{c}\text { Observed } \\ \text { inhibition } \\ (\%)\end{array}}^{\text {Inhibition of growth rate }} \underbrace{\text { g. }}_{\begin{array}{c}\text { Sum of individual } \\ \text { inhibitions } \\ (\%)\end{array}}$

(a) NCM alone as the basal medium

\begin{tabular}{|c|c|c|}
\hline O.OI mM-deGlc & I5. I & - \\
\hline $0.04 \mathrm{mM}$-deGlc & 44.9 & - \\
\hline $0.10 \mathrm{mM}-\mathrm{GA}$ & $14 \cdot 6$ & - \\
\hline $0.25 \mathrm{~mm}-\mathrm{GA}$ & $80 \cdot 6$ & - \\
\hline $0.20 \mathrm{~mm}-\mathrm{Sor}$ & $6 \cdot 7$ & - \\
\hline $0.80 \mathrm{~mm}-\mathrm{Sor}$ & $42 \cdot 6$ & - \\
\hline $0.20 \mathrm{~mm}$-Sor $+0.01 \mathrm{~mm}$-deGlc & $19 \cdot 0$ & $2 \mathrm{I} \cdot 8$ \\
\hline $0.20 \mathrm{~mm}$-Sor $+0.04 \mathrm{~mm}$-deGlc & $44 \cdot 6$ & $5 \mathrm{I} \cdot 6$ \\
\hline $0.20 \mathrm{mM}-\mathrm{Sor}+0.10 \mathrm{mM}-\mathrm{GA}$ & $8 \cdot 0$ & $2 \mathrm{I} \cdot 3$ \\
\hline $0.20 \mathrm{mM}-\mathrm{Sor}+0.25 \mathrm{mM}-\mathrm{GA}$ & $84 \cdot 4$ & $87 \cdot 3$ \\
\hline $0.80 \mathrm{~mm}$-Sor $+0.01 \mathrm{~mm}$-deGlc & 15.6 & $57 \cdot 7$ \\
\hline 0.80 mM-Sor +0.04 mM-deGlc & $48 \cdot 0$ & 87.5 \\
\hline $0.80 \mathrm{mM}-\mathrm{Sor}+0.10 \mathrm{mM}-\mathrm{GA}$ & $60 \cdot 3$ & $57 \cdot 2$ \\
\hline $0.80 \mathrm{mM}-\mathrm{Sor}+0.25 \mathrm{mM}-\mathrm{GA}$ & $92 \cdot \mathrm{I}$ & $123 \cdot 2$ \\
\hline $0.10 \mathrm{mM}-\mathrm{GA}+0.0 \mathrm{I} \mathrm{mm}-\mathrm{deGlc}$ & $34 \cdot 3$ & 29.7 \\
\hline $0.10 \mathrm{~mm}-\mathrm{GA}+0.04 \mathrm{mM}-\mathrm{deGlc}$ & $4 \mathrm{I} \cdot 4$ & $59 \cdot 5$ \\
\hline $0.25 \mathrm{~mm}-\mathrm{GA}+0.01 \mathrm{mM}-\mathrm{deGlc}$ & $89 \cdot 4$ & 95.7 \\
\hline $0.25 \mathrm{~mm}-\mathrm{GA}+0.04 \mathrm{mM}-\mathrm{deGlc}$ & $74 \cdot 3$ & 125.5 \\
\hline
\end{tabular}

(b) $\mathrm{NCM}+5$ mM-glucose as the basal medium

\begin{tabular}{|c|c|c|}
\hline $0.5 \mathrm{~mm}$-deGlc & 23.9 & - \\
\hline $4.0 \mathrm{mM}$-deGlc & $52 \cdot 4$ & - \\
\hline $6.0 \mathrm{mM}-\mathrm{GA}$ & $23 \cdot 2$ & - \\
\hline I5.0 mM-GA & $85 \cdot 5$ & - \\
\hline $2.0 \mathrm{mM}-\mathrm{Sor}$ & $15 \cdot 8$ & - \\
\hline $50.0 \mathrm{mM}-\mathrm{Sor}$ & $38 \cdot 3$ & - \\
\hline $2.0 \mathrm{mM}$-Sor $+0.5 \mathrm{mM}$-deGlc & $29 \cdot 0$ & $39 \cdot 7$ \\
\hline $2.0 \mathrm{mM}-\mathrm{Sor}+4.0 \mathrm{mM}-\mathrm{deGlc}$ & $64 \cdot 0$ & $68 \cdot 2$ \\
\hline $2 \cdot 0 \mathrm{mM}-\mathrm{Sor}+6 \cdot 0 \mathrm{mM}-\mathrm{GA}$ & $30 \cdot 8$ & $39 \cdot 0$ \\
\hline $2.0 \mathrm{mM}-\mathrm{Sor}+\mathrm{I} 5.0 \mathrm{mM}-\mathrm{GA}$ & $79 \cdot 7$ & IOI $\cdot 3$ \\
\hline $50.0 \mathrm{mM}$-Sor $+0.5 \mathrm{mM}$-deGlc & $39 \cdot 0$ & $62 \cdot 2$ \\
\hline $50.0 \mathrm{mM}-\mathrm{Sor}+4.0 \mathrm{mM}-\mathrm{deGlc}$ & $65 \cdot I$ & $90 \cdot 7$ \\
\hline $50.0 \mathrm{mM}-\mathrm{Sor}+6.0 \mathrm{mM}-\mathrm{GA}$ & 43.9 & $61 \cdot 5$ \\
\hline $50.0 \mathrm{mM}-\mathrm{Sor}+15.0 \mathrm{mM}-\mathrm{GA}$ & $80 \cdot 2$ & I 23.8 \\
\hline $6.0 \mathrm{mM}-\mathrm{GA}+0.5 \mathrm{mM}-\mathrm{deGlc}$ & $23 \cdot 0$ & $47 \cdot 1$ \\
\hline $6.0 \mathrm{mM}-\mathrm{GA}+4.0 \mathrm{mM}-\mathrm{deGlc}$ & $50 \cdot 6$ & $75 \cdot 6$ \\
\hline I $5.0 \mathrm{~mm}-\mathrm{GA}+0.5 \mathrm{~mm}$-deGlc & $73 \cdot 5$ & $109 \cdot 4$ \\
\hline I $5.0 \mathrm{mM}-\mathrm{GA}+4.0 \mathrm{mM}-\mathrm{deGlc}$ & $76 \cdot 9$ & 137.9 \\
\hline
\end{tabular}

few scattered compartments still having their contents intact. The apical compartments were much more sensitive to the inhibitors if the medium contained fructose or acetate than if it contained glucose.

\section{DISCUSSION}

The various effects of the three hexose analogues are similar to effects demonstrated in many other organisms. The major difference between this and previous work lies in the clear indication that the separate inhibitions were very closely related to one another. Comparison with work described in the literature is made difficult by the diversity of organisms used and by the very fact that the agents have been considered in isolation.

It is unlikely that the effects of these analogues on Coprinus lagopus can be ascribed to any serious inhibition of sugar uptake since the greatest degrees of inhibition of extension 

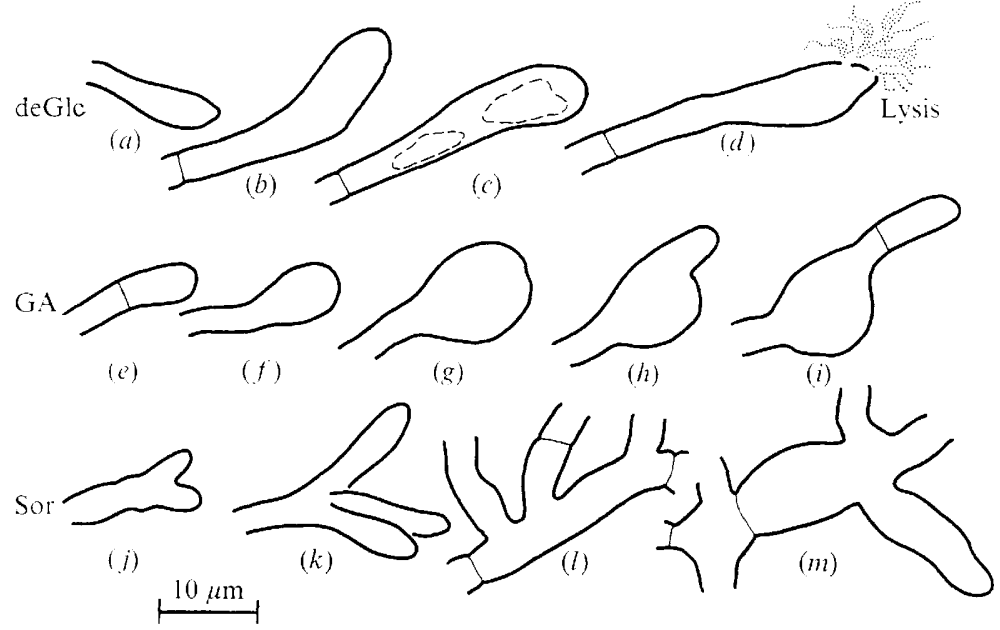

Fig. 4. Shapes assumed by terminal and intercalary compartments of hyphae grown on media containing inhibitory concentrations of hexose analogues. Drawings are tracings from photographs of individual hyphal compartments observed with oil immersion objectives. The figures are arranged in a probable developmental series but individual hyphae have not been followed through the development of these aberrations. Note the vacuolation and eventual lysis of swollen terminal cells which occurred on deGlc; the extreme swelling of terminal compartment on GA; and the frequent branching and giant mycelial cells seen on Sor. The following media were used: $(a),(b),(d), 5 \mathrm{~mm}-$ Glc + 20 mM-deGlc; $(c), 5$ mM-Ace + o. I mM-deGlc; $(e), 5$ mM-Glc + I 5 mM-GA; $(f),(h), 5$ mMFru + 0.2 mM-GA; $(g),(i), 40$ mM-Glc + 80 mM-GA; $(j),(m), 5$ mM-Ace + 2 mM-Sor; $(k),(l), 5$ mM-Glc + 75 mM-Sor.

rate were observed on media which either lacked any normal carbon source or which contained acetate as a normal carbon source. Preliminary experiments on the removal of sugars from the suspending medium by oidia support this conclusion. For example, a fructose: deGlc ratio which completely inhibited extension growth was found to inhibit fructose uptake by only $7 \%$. Consequently, the most important inhibitory actions must be exerted after the analogue and the sugar have entered the hypha.

That acetate and fructose were the least able to combat the analogues suggests important inhibitory actions in the pathways of carbohydrate biogenesis and fructose metabolism. Serious inhibitions of fructose diphosphatase and phosphohexose isomerase might therefore be suspected. Considerable activity against an isomerase may also be deduced from the fact that mannose was uniformly less able than glucose to reverse the inhibitions. Alternatively the sensitivity differentials may reflect the different abilities of the normal hexoses to inhibit analogue uptake. In this context it should be noted that sensitivity differentials of the magnitude demonstrated here are very unusual in the filamentous fungi (Moore, I969a).

Figure 2 shows that the inhibitions on acetate and fructose media were fundamentally different in kinetic terms from those which occurred on mannose and glucose media.

A close relationship between the inhibitors is indicated by the data of Table 3 and by the similarities between the morphological aberrations each inhibitor caused. The data of Table 3 might be explicable in terms of competition between the analogues for transport into the cell. While this remains a possibility to be tested it seems that the analogues did not seriously affect the uptake of normal sugars so it may well be that significant analogue interaction did not occur at this level either. The fact remains, though, that at some level the analogues did compete with one another, suggesting at least one common point of action. 
There are thus quite definite indications of significant inhibitory activities, by all three analogues, at early stages in carbohydrate metabolism. Sites of action so early in glycolysis, however, cannot directly explain the gross morphological aberrations the agents have been shown to cause. These aberrations are presumably related to other effects which the agents, or their derivatives, exert on the formation of the hyphal walls. It is certainly established that deGlc seriously affects wall synthesis in yeasts. This is undoubtedly the basis of the fungicidal action that deGlc has on them since only growing cells were killed (Heredia et al. 1964) and yeast wall fragments were found in the treated suspensions (Megnet, 1965). More specifically, the action appears to result from an initial trapping of uridine nucleotides by deGlc-6-P and a consequent blockage of polysaccharide synthesis (Heredia et al. 1964; Biely \& Bauer, I966; Farkas, Svoboda \& Bauer, 1969) with the result that lysis occurs at points coinciding with the regions of growth of the glucan layers of the cell wall (Johnson, 1968). Although such detailed analysis has not yet been attempted in Coprinus there is an obvious analogy in that deGlc caused lysis of active hyphal tip compartments so that it is not unlikely that similar biochemical events were in operation.

Close analogies also exist between the effects of Sor on Coprinus lagopus and its effects on Neurospora. When grown on medium containing Sor the normally rapidly spreading growth habit of Neurospora becomes changed to the so-called colonial form. The growth rate is greatly reduced, cells are much shortened, branching frequency is increased with the frequent and almost completely abnormal occurrence of branched tips (Tatum, Barratt \& Cutter, 1949). This description could equally well apply to $C$. lagopus. In Neurospora the morphological changes were shown to be accompanied by changes in the chemical composition of the hyphal walls. The ratio of glucosamine to glucose in wall hydrolysates was more than doubled (de Terra \& Tatum, I96I, I963) although there did not appear to be any large-scale incorporation of sorbose.

The conclusion thus seems inevitable that deGlc, Sor and, by implication, GA cause critical alterations in the composition and construction of fungal walls. The observations also imply some effect on the pattern of morphogenesis in hyphae. Butler (196I) showed that extension growth rate of hyphae of Coprinus disseminatus was positively correlated with the diameter of the hypha; broadly speaking the greater was the diameter of a hypha the faster was its rate of extension growth. Yet it can be seen from the experiments described above that Sor in particular, and deGlc and GA to lesser extents, reversed this relationship, causing both a decrease in growth rate and a swelling of hyphal compartments. Thus the analogues were capable of interfering with a basic parameter of hyphal morphogenesis.

The drawings of analogue-treated hyphae shown in Fig. 4 are strikingly similar to the figures of hyphal tips of Fusarium and Neurospora treated with water and hyper- and hypotonic solutions given by Robertson (1958, 1959). Robertson suggested an explanation based on osmotic effects; the mechanism of extension growth being supposed to be sensitive to rapid changes in the rate and direction of water flow through the cell membrane. Fig. 4 shows, for example, that extreme swelling of terminal cells occurred on a medium containing $40 \mathrm{~mm}$-glucose $+80 \mathrm{~mm}-\mathrm{GA}$ and this might lead to the suspicion that the effect was basically osmotic in nature. However, much the same degree of swelling was observed in hyphae grown on $5 \mathrm{~mm}$-fructose $+0 \cdot 2 \mathrm{mM}$-GA. Thus the effect must have been related to the molecule itself; its concentration was of importance only in relation to the concentrations and identities of other molecules which had some influence on the inhibition mechanism. The implication is that the processes which control the shape of the compartment must be very closely related to the processes which determine the chemical composition of the hyphal wall. 
The authors are indebted to $\mathrm{Mr} \mathrm{H}$. Waters for his assistance with parts of this work. It is again a pleasure to acknowledge the skilled technical assistance of Miss Elizabeth Callan.

\section{REFERENCES}

Barnett, H. L. \& Lilly, V. G. (195I). The inhibitory effects of sorbose on fungi. Science, New York Ir4 439-440.

BIELY, P. \& BAUER, S. (1966). The formation of uridine diphosphate-2-deoxy-D-glucose in yeast. Biochimica et biophysica acta 121, 213-214.

BiLgrami, K. S. (1963). The utilization of L-sorbose by some pathogenic species of Phyllosticta. Proceedings of the Indian Academy of Science B 57, 230-234.

ButLer, G. M. (1961). Growth of hyphal branching systems in Coprinus disseminatus. Annals of Botany 25, 34I-352.

Farkas, V., Svoboda, A. \& BaUeR, S. (1969). Inhibitory effect of 2-deoxy-D-glucose on the formation of the cell wall in yeast protoplasts. Journal of Bacteriology 98, 744-748.

Heredia, C. F., De LA Fuente, G. \& Sols, A. (1964). Metabolic studies with 2-deoxyhexoses. I. Mechanisms of inhibition of growth and fermentation in bakers yeast. Biochimica et biophysica acta 86, 216-223.

Johnson, B. F. (1968). Lysis of yeast cell walls induced by 2-deoxyglucose at their sites of glucan synthesis. Journal of Bacteriology 95, II 69-I 172.

LiLLY, V. G. \& BARNETT, H. L. (1953). The utilization of sugars by fungi. Bulletin of the West Virginia Agricultural Experiment Station 362T-58

MANDels, G. R. (1965). Kinetics of fungal growth. In The Fungi, vol. I, pp. 599-612. London: Academic Press.

Matsushima, T. \& Klug, R. J. (1958). Utilization of L-sorbose by monosporidial lines and mutants of Ustilago maydis. American Journal of Botany 45, $165-168$.

MEGNET, R. (1965). Effect of 2-deoxyglucose on Schizosaccharomyces pombe. Journal of Bacteriology 90 , 1032-1035.

MoOrE, D. (1968). The effect of 2-deoxy-D-glucose on the growth and respiration of Coprinus lagopus. Journal of General Microbiology 52, 433-439.

MOORE, D. (I969a). Effect of 2-deoxy-D-glucose on mycelial growth of filamentous fungi. Transactions of the British Mycological Society 53, I39-I $4 \mathrm{I}$.

MOORE, D. (1969b). Sources of carbon and energy used by Coprinus lagopus sensu Buller. Journal of General Microbiology 58, 49-56.

Opdoux, L. (1952). Utilization comparée du sorbose et du fructose naturels par une centaine d'Homobasidiomycétes. Comptes rendus de l'Academie des sciences, Paris 234, 654-657.

RoBerTSON, N. F. (1958). Observations on the effect of water on the hyphal apices of Fusarium oxysporum. Annals of Botany 22, $159-173$.

ROBERTSON, N. F. (1959). Experimental control of hyphal branching and branch form in hyphomycetous fungi. Journal of the Linnean Society 56, 207-2 I I.

Tatum, E. L., Barratt, R. W. \& CutTer, V. M. (I949). Chemical induction of colonial paramorphs in Neurospora and Syncephalastrum. Science, New York ro9, 509-51 I.

DE TeRra, N. \& TATUM, E. L. (196I). Colonial growth of Neurospora crassa. Science, New York 134, 10661068.

DE TeRra, N. \& TATUM, E. L. (1963). A relationship between cell wall structure and colonial growth in Neurospora crassa. American Journal of Botany 50, 669-677.

TrincI, A. P. J. (1969). A kinetic study of the growth of Aspergillus nidulans and other fungi. Journal of General Microbiology 57, $1 \mathrm{I}-24$.

TRINCI, A. P. J. \& GULL, K. (1970). Effect of actidione, griseofulvin and triphenyltin acetate on the kinetics of fungal growth. Journal of General Microbiology 6o, 287-292. 